Supporting Information

\title{
A Surface Coating Approach to Overcome Mucosal Entrapment of DNA Nanoparticles for Oral Gene Delivery of Glucagon-like Peptide
}

\section{1}

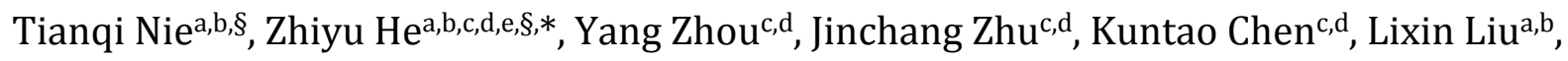
Kam W. Leong ${ }^{\mathrm{a}, \mathrm{b}, \mathrm{g}}$, Hai-Quan Mao $\mathrm{Ma}^{\mathrm{a}, \mathrm{c}, \mathrm{d}, \mathrm{e}, \mathrm{f}, * \text {, and Yongming Chen }}{ }^{\mathrm{a}, \mathrm{b}, *}$

aSchool of Materials Science and Engineering, Sun Yat-sen University, Guangzhou 510275, China

${ }^{\mathrm{b}}$ Center for Functional Biomaterials, and Key Laboratory for Polymeric Composite and Functional Materials of Ministry of Education, Sun Yat-sen University, Guangzhou 510275, China

'Department of Materials Science and Engineering, Whiting School of Engineering, Johns Hopkins University, Baltimore, MD 21218, USA

dInstitute for NanoBioTechnology, Johns Hopkins University, Baltimore, MD 21218, USA

eTranslational Tissue Engineering Center, Johns Hopkins University School of Medicine, Baltimore, MD 21287, USA

fDepartment of Biomedical Engineering, Johns Hopkins University School of Medicine, Baltimore, MD 21287, USA

gDepartment of Biomedical Engineering, Columbia University, New York, NY 10027, USA

$\S$ These authors contributed equally to this work.

\section{*To whom correspondence should be addressed:}

Yongming Chen, School of Materials Science and Engineering, Sun Yat-sen University, No. 135, Xingang Xi Road, Guangzhou 510275, China; E-mail: chenym35@mail.sysu.edu.cn; Hai-Quan Mao, INBT, 100 Croft Hall, 3400 N. Charles Street, Baltimore, MD 21218, U.S.A.; E-mail: hmao@jhu.edu; 
Zhiyu He, INBT, 100 Croft Hall, 3400 N. Charles Street, Baltimore, MD 21218, U.S.A.; E-mail: zhe19@jhu.edu. 
A

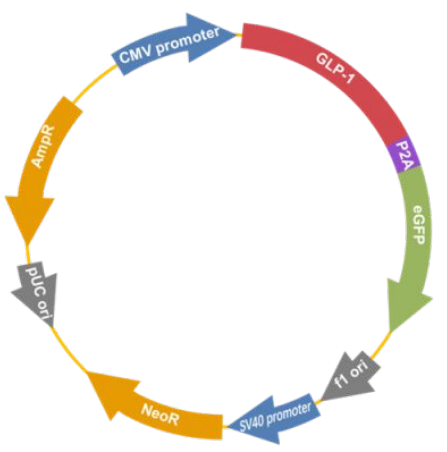

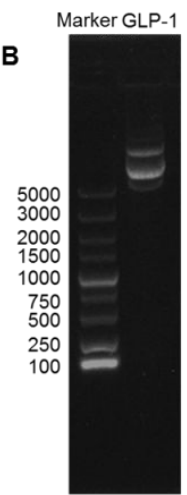

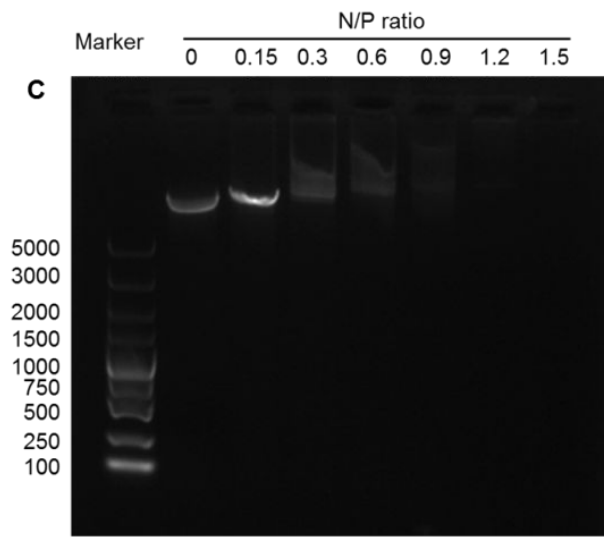

Figure S1. Characterization of the plasmid constructed to encode GLP-1. (A) The construction of plasmid encoding GLP-1. (B) The gel electrophoresis image of plasmid encoding GLP-1. (C) Gel retardation assay of IPEI/DNA nanoparticles at various N/P ratios. 


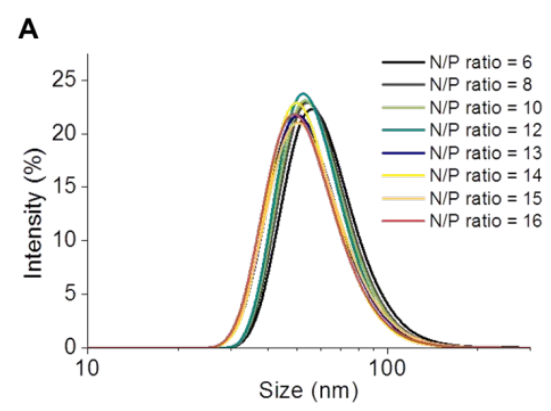

B

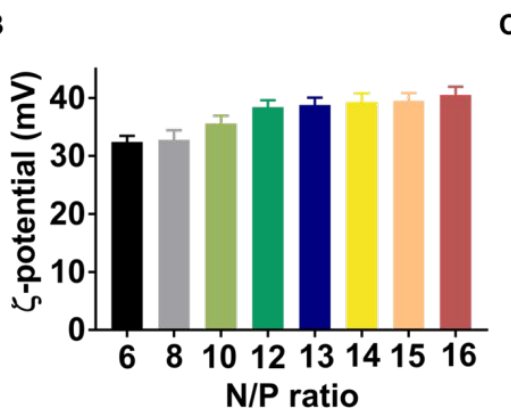

C

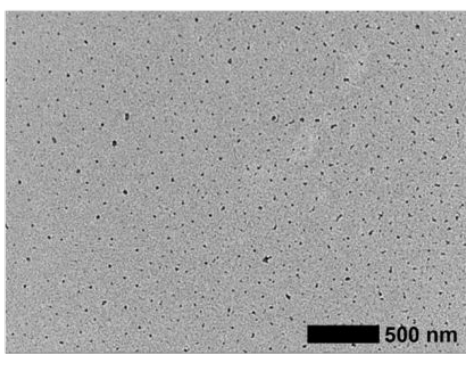

Figure S2. Characterization of IPEI-DNA nanoparticle core (NP-1). (A) The size distribution of IPEI/DNA nanoparticles with different N/P ratio.

(B) The zeta potential of $I$ PEI/DNA nanoparticles with different N/P ratio. (C) The TEM image of $/$ PEI-DNA nanoparticles. Data was shown as mean \pm S.D $(n=3)$. 
A

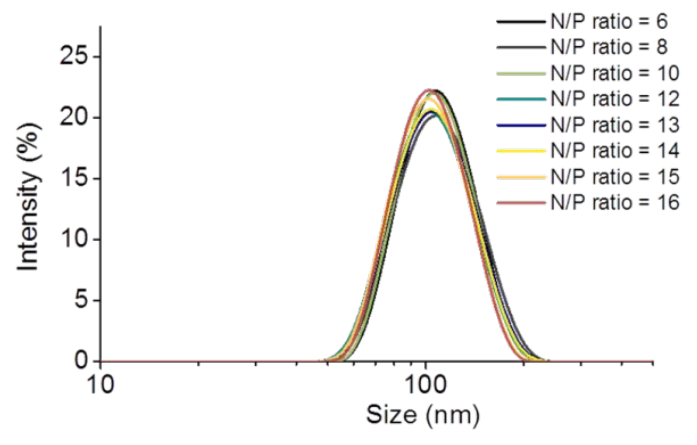

B

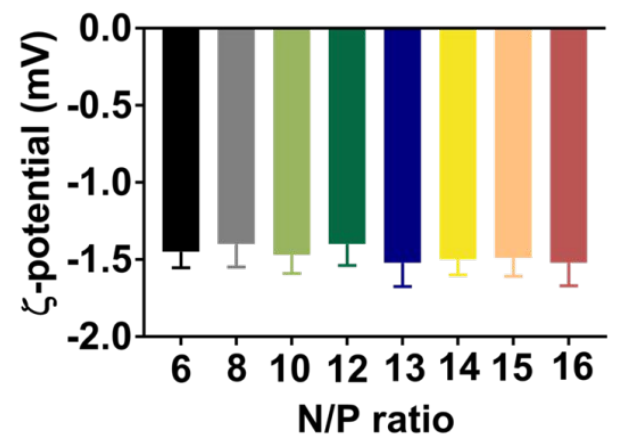

Figure S3. Characterization of DPPC/DMG-PEG/(IPEI-DNA) nanoparticle (NP-3). (A) The size distribution of NP-3 with different N/P ratio. (B) The zeta potential of NP-3 with different N/P ratio. Data was shown as mean \pm S.D $(n=3)$. 


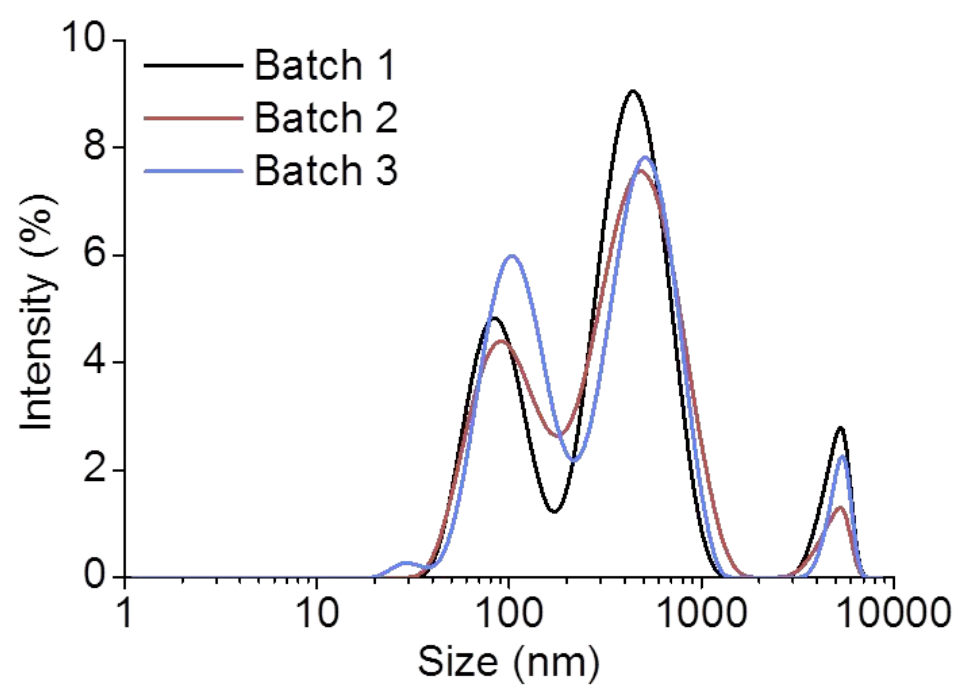

Figure S4. Size distribution of DPPC/DMG-PEG/(IPEI/DNA) N/Ps by traditional method. 


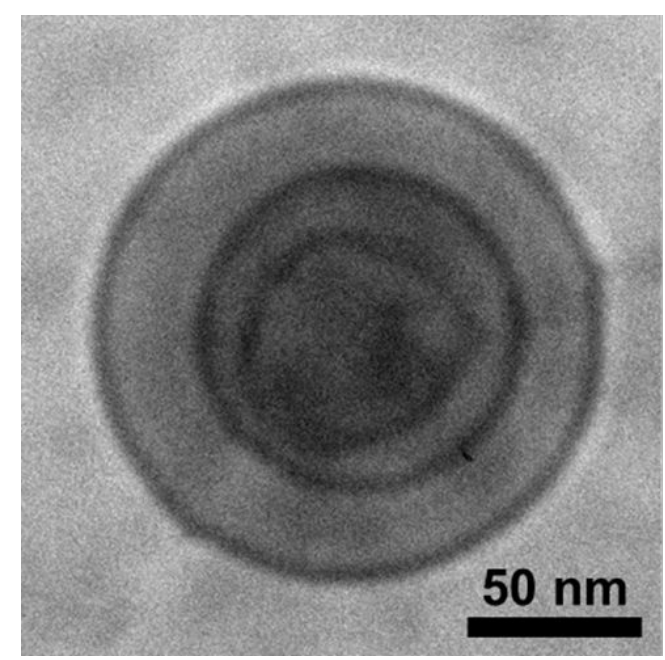

Figure S5. The cryo-TEM image of one DPPC/DMG-PEG/(IPEI/DNA) nanoparticles. 


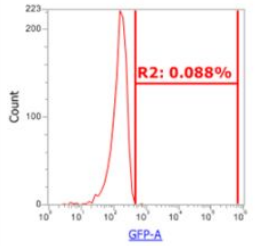

Control

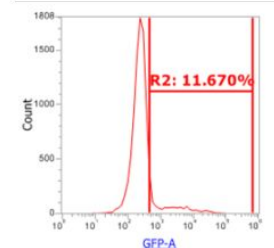

6

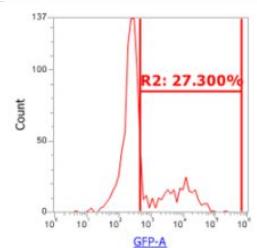

8

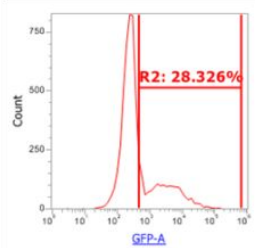

10

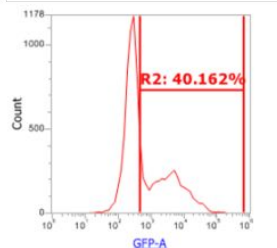

12 N/P ratio
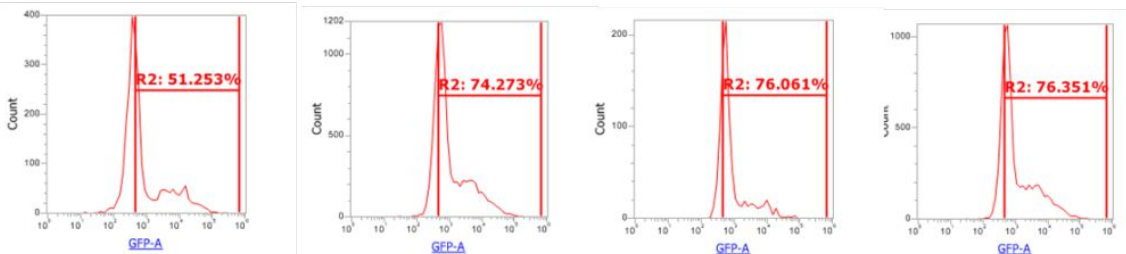

13

14

15 16

Figure S6. Flow cytometric analysis of 293T cells treated with DPPC/DMG-PEG/(PEI-DNA) nanoparticles prepared at different N/P ratios. 


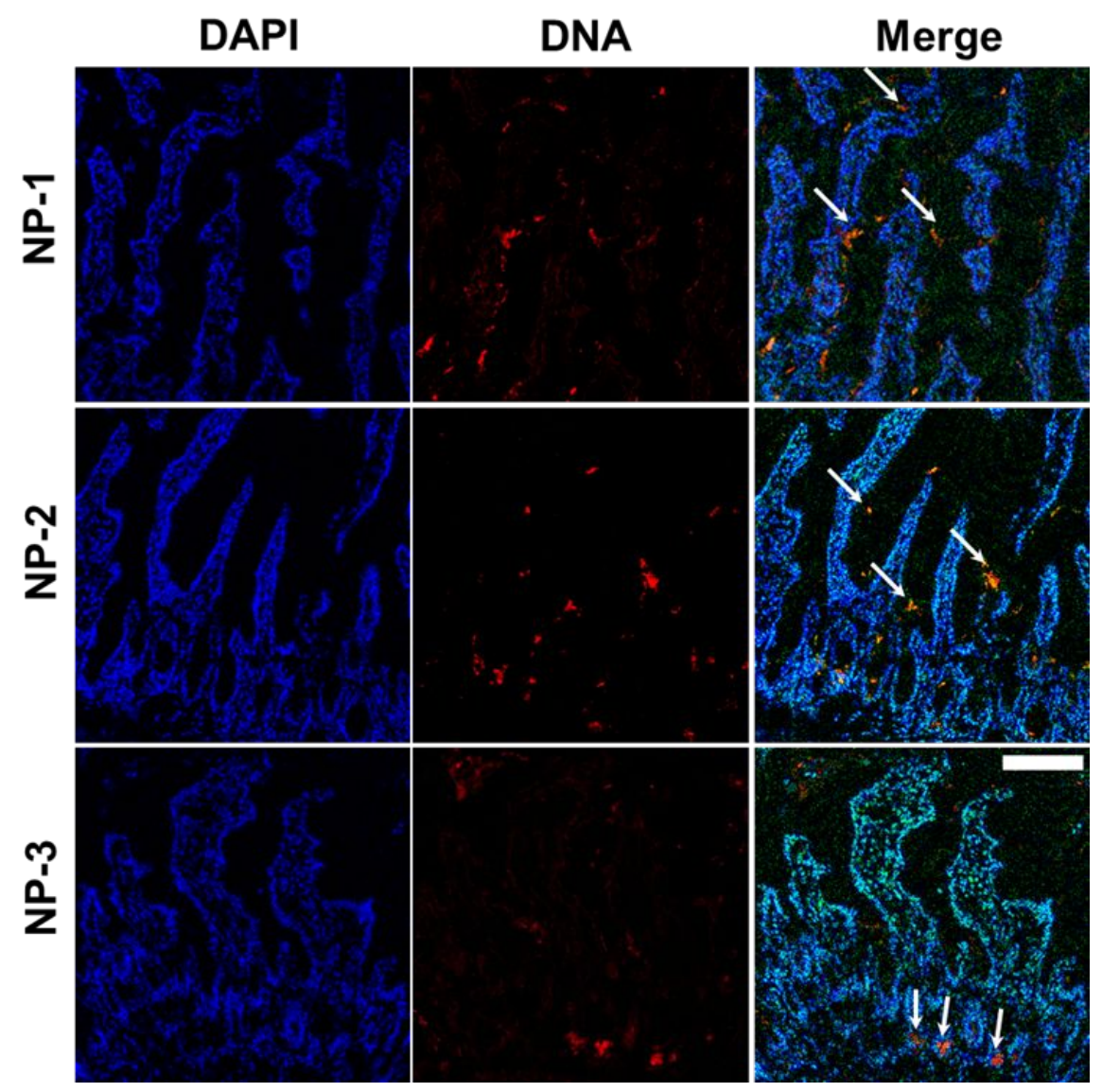

Figure S7. The transport property of DPPC/DMG-PEG/(PEI-DNA) nanoparticles crossing the mucus layer in vivo. (Scale bar $=100 \mu \mathrm{m})$ 

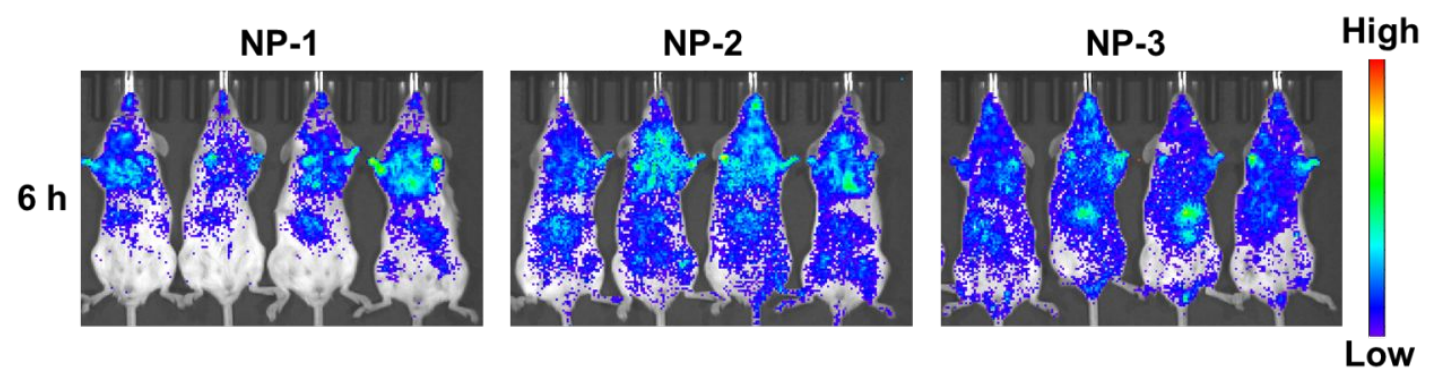

Figure S8. Whole-body bioluminescence imaging of Balb/c mice at $6 \mathrm{~h}$ post administration with single dosage of NP-1, NP-2 or NP-3 containing plasmid DNA encoding luciferase. 


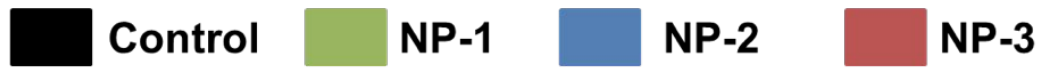

A

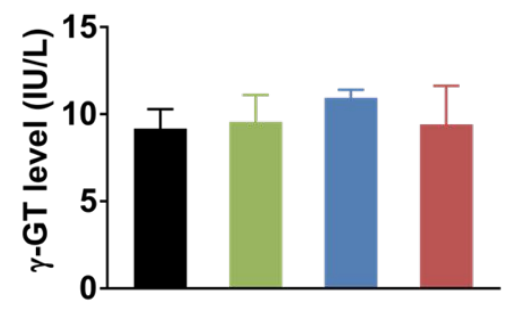

B

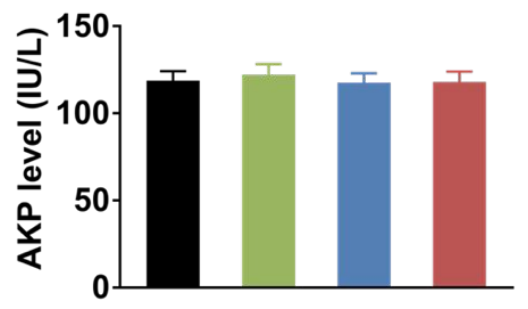

Figure 59. The enzyme activities of (A) $\gamma$-GT and (B) AKP from different treatment groups after the oral dosing. Error bars indicate S.D. $(n=3)$. 
Table S1. The stability of the coated DNA nanoparticles in different $p H$ environments

\begin{tabular}{cccc}
\hline Nanoparticle & $\begin{array}{c}\text { Incubation time } \\
\text { (min) }\end{array}$ & $\begin{array}{c}\text { Average size in } \\
\text { HCl solution (pH 2.5) } \\
\text { (nm) }\end{array}$ & $\begin{array}{c}\text { Average size in } \\
\text { PBS (pH 7.4) } \\
(\mathrm{nm})\end{array}$ \\
\hline NP-2 & 5 & $74.3 \pm 1.5$ & $83 \pm 1.8$ \\
& 15 & $73.5 \pm 1.9$ & $96.2 \pm 1.7$ \\
& 30 & $72.7 \pm 1.6$ & $87.7 \pm 1.7$ \\
& 60 & $71.5 \pm 1.3$ & $81.5 \pm 1.4$ \\
& 120 & $85.4 \pm 1.8$ & $87.2 \pm 1.5$ \\
& 240 & $95.8 \pm 2.1$ & $89.5 \pm 1.3$ \\
\hline NP-3 & 0 & & $103 \pm 2.1$ \\
& 5 & $94.4 \pm 2.4$ & $104 \pm 2.3$ \\
& 15 & $93.4 \pm 2.2$ & $98.8 \pm 1.8$ \\
& 30 & $91.3 \pm 1.9$ & $100.5 \pm 2.6$ \\
& 60 & $84.1 \pm 2.3$ & $96.2 \pm 2.5$ \\
& 120 & $85.7 \pm 2.2$ & $97.2 \pm 2.3$ \\
& 240 & $86.5 \pm 1.9$ & \\
\hline
\end{tabular}

Note: Data are shown as mean \pm S.D. $(n=3)$.

Table S2. Relative light units (RLU) measuring the relative transgene expression of luciferase transgene in different organs at 24 post-oral administration

\begin{tabular}{cccc}
\hline Nanoparticle & $\begin{array}{c}\text { Liver } \\
\text { (RLU/mg tissue) }\end{array}$ & $\begin{array}{c}\text { Lung } \\
\text { (RLU/mg tissue) }\end{array}$ & $\begin{array}{c}\text { Small intestine } \\
\text { (RLU/mg tissue) }\end{array}$ \\
\hline NP-1 & 200.35 & 186.54 & 33.14 \\
NP-2 & 404.27 & 284.37 & 40.55 \\
NP-3 & 644.25 & 448.46 & 80.56 \\
\hline
\end{tabular}

